and so we did. In the end, the Academic Board accepted our proposal. The program runs on students in the fourth Academic Year, in small groups, and on the premises of the school. The other one with postgraduate Medical Students didn't accept the proposal of our program, with the justification that as a department of a foreign Medical school, they couldn't implement anything in the students program. So far, more than 10 trainings have been conducted. At the end of the first year that the program was implemented, the Medical School asked from the organisation for a Patient Instructor to take part at the Musculoskeletal Examination and be a model for the Examination. Seeing our success, and taking into consideration the students' evaluation of the program, especially the fact that $95 \%$ of them said, 'It was very important for them to have a Patient Expert Program during their studies' our organisation decided to expland our collaboration with more Healthcare Professions Universities. Our original goal had been to collaborate with one of the medical schools/universities, but now we have bigger plans. Now we want this program to be established in all the universities in the country with the Patient Expert RMD's program implemented in all health professional curriculums.

Conclusion: The importance of the Patient Expert in RMD's is unquestionable. Patient Expert Program is an excellent tool to raise awareness on RMD's. Also it is very helpful for the Medical or Health care Professions student as it can help them better understand the disease and the patients. The success of the program is also giving motivation to the organisation to expand and improve the program. It also shows the significance of the program for the next generation of Physicians and Healthcare Professionals. Our organisation is leading in patient centered care and is proof that making the patient the most important is the target of treatment for RMD's in our country.

Disclosure of Interests: None declared

DOI: 10.1136/annrheumdis-2020-eular.2111

\section{OP0259-PARE WORLD YOUNG RHEUMATIC DISEASES (WORD) DAY: THE FIRST INTERNATIONAL AWARENESS DAY FOR PAEDIATRIC RHEUMATIC DISEASES}

S. Stones ${ }^{1}$, E. Smith ${ }^{2}$, S. Ainsworth ${ }^{1}$, V. Buys ${ }^{1}$, W. Costello ${ }^{1}$, Y. Egert ${ }^{1}$ H. Foster ${ }^{3}$, L. Lamot ${ }^{4}$, B. J. Prakken ${ }^{5}$, C. Scott ${ }^{6}$. ${ }^{1}$ European Network for Children with Arthritis, Geneva, Switzerland; ${ }^{2}$ University of Liverpool, Department of Women's and Children's Health, Liverpool, United Kingdom; ${ }^{3}$ Newcastle University Medicine Malaysia, Johor Bahru, Malaysia; ${ }^{4}$ Sestre Milosrdnice University Hospital Center and University of Zagreb School of Medicine, Department of Pediatrics, Zagreb, Croatia; ${ }^{5}$ University Medical Center Utrecht, Department of Pediatric Immunology, Utrecht, Netherlands; ${ }^{6}$ University of Cape Town/Red Cross War Memorial Children's Hospital, Paediatric Rheumatology, Cape Town, South Africa

Background: There is a lack of awareness of paediatric rheumatic diseases (PRDs), among the public, and certain groups of healthcare professionals, such as general practitioners [1]. To help improve awareness and understanding of PRDs, World Young Rheumatic Diseases Day (WORD Day) was established in 2019.

Objectives: The aim of WORD Day, which took place on 18 March 2019, was to raise awareness of PRDs, while informing young people, families, healthcare professionals, teachers, and the public about the importance of timely referral plus early diagnosis and access to appropriate treatment and support.

Methods: A steering committee was established for the inaugural campaign. An external agency was appointed to provide digital support, with an official social media campaign launching in December 2018. Social media analytics were used to measure the impact of official social media platforms. A range of branded materials were also made available (Fig 1), including an official campaign video. In addition, a specific engagement activity (\#ButtonChallenge2019) was launched. This challenged participants to button up a piece of clothing while wearing gloves, to simulate the difficulties that young people with PRDs face undertaking daily activities. As part of the campaign launch, a list of suggested activities were provided, along with template press releases.

Results: Several face-to-face and virtual events took place globally on or around WORD Day 2019, with 34 countries reporting events (Fig 2). Examples of events included lectures, social gatherings and media appearances. An official WORD Day tweet chat was also hosted. Regarding social media impact, between December 2018 and March 2019, a total of 2,585 and 660 individuals followed the official Facebook and Twitter accounts, respectively. Facebook posts were seen 646,000 times since the start of the campaign, with over 60,000 of Facebook reach from posts published on 18 March 2019 exclusively. A total of 270,800 impressions were observed on Twitter. The official \#WORDDay2019 hashtag was seen by 533,955 unique accounts on 18 March 2019 alone, with 3,334,699 impressions. Posts with the hashtag were retweeted 1,112 times on WORD Day, with a total of 1,568 tweets recorded that day. With regards to the \#ButtonChallenge2019, the challenge video was viewed for 6,700 minutes and received participation from across the world. Compared to the industry benchmark, the average engagements per post for WORD Day-related content was significantly higher compared to other medical and non-for-profit social media pages.

Conclusion: WORD Day 2019 was the first international campaign focused solely on PRDs. Organic and paid social media content aided the dissemination of the WORD Day message, with Facebook proving to be the most popular social platform. Despite a wealth of different content published, authentic materials, namely video content, proved to be the most popular with users, particularly when it featured material designed by and with young people with PRDs. It was demonstrated that awareness events can often be resource-light and easily implemented across a range of diverse countries. It is anticipated that the global reach of WORD Day will increase over time as the campaign becomes more established.

References:

[1] Egert $Y$ et al. Children and young people get rheumatic disease too. The Lancet Child \& Adolescent Health. 2019;3(1):8-9.
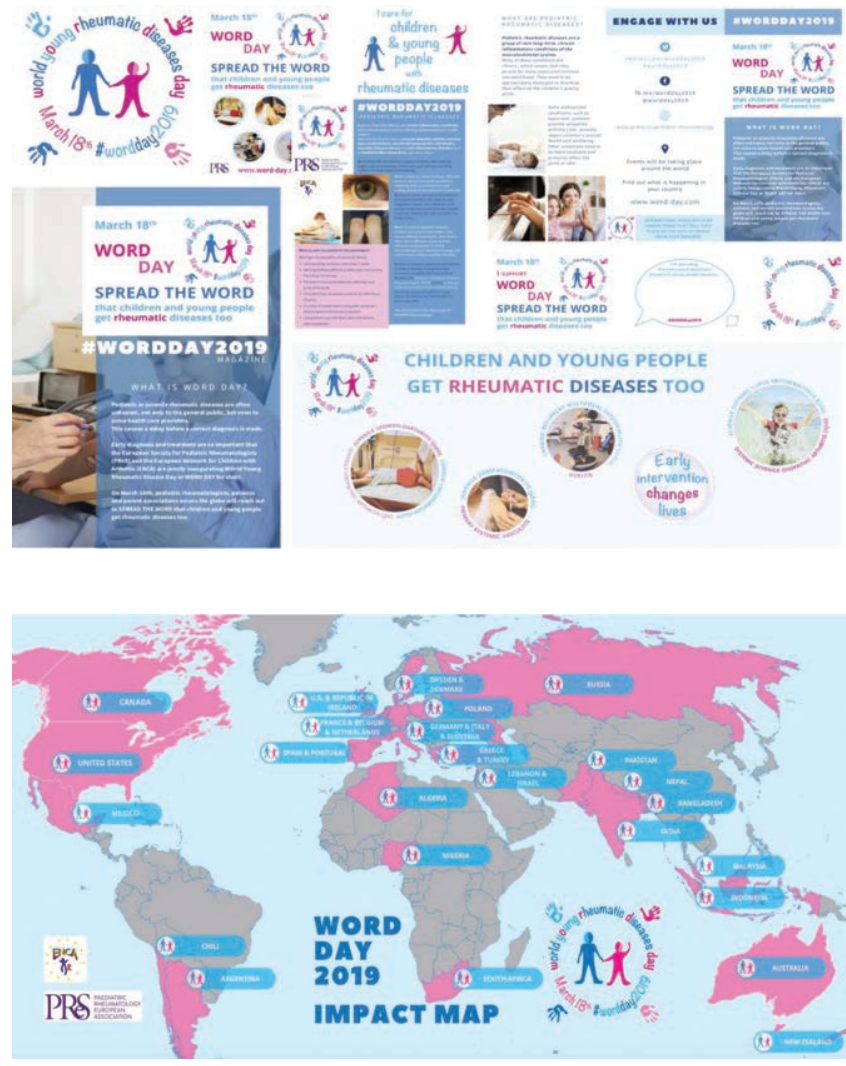

Acknowledgments: PReS for their financial support.

Disclosure of Interests: Simon Stones Consultant of: I have been a paid consultant for Envision Pharma Group and Parexel. This does not relate to this abstract. Speakers bureau: I have been a paid speaker for Actelion and Janssen. These do not relate to this abstract., Eve Smith: None declared, Sammy Ainsworth None declared, Veerle Buys: None declared, Wendy Costello: None declared Yona Egert: None declared, Helen Foster: None declared, Lovro Lamot: None declared, Berent J. Prakken: None declared, Christiaan Scott: None declared DOI: 10.1136/annrheumdis-2020-eular.1794

\section{OP0260-PARE EXPERIENCE EXCHANGE - FUNDRAISING AND PATIENT EXPERT PROGRAM}

\section{N. Nedić ${ }^{1}$ ' Serbia, Belgrade, Serbia}

Background: Since the establishment of ORS, we have been organising different activities for rheumatic diseases patients and their supporters within our five branches. After celebrating our $10^{\text {th }}$ anniversary in 2017 we were keen to expand and improve our activities, learning from an organisation with long tradition, in order to strengthen our position and to start reaching another level - growing from a small to a medium-sized organization in the next three years.

The main source of our income were the pharmaceutical companies (94\%). We wanted to reduce their contribution to our budget by $20 \%$ in the next three years. We had established good cooperation with two medical high schools and we would like to consider cooperation with a medical faculty. We were keen to gain insight into the Patient Expert Programme (PEP) project that Swedish Rheumatism Association had developed and hoped to then begin and steadily develop 